\title{
Pattern Deformities and Cell Loss in Engrailed-2 Mutant Mice Suggest Two Separate Patterning Events during Cerebellar Development
}

\author{
Barbara Kuemerle, ${ }^{1}$ Hadi Zanjani, ${ }^{2,4}$ Alexandra Joyner, ${ }^{3}$ and Karl Herrup ${ }^{1}$ \\ ${ }^{1}$ Alzheimer Research Laboratory, Case Western Reserve Medical School, Cleveland, Ohio 44106, 2Laboratoire de \\ Neurobiologie du Developpement, Institut des Neuroscience (Centre National de la Recherche Scientifique Unité de \\ Recherche Associée 1488), Université Pierre \& Marie Curie, 75005 Paris, France, ${ }^{3}$ Skirball Institute of Biomolecular \\ Medicine, New York University Medical Center, New York, New York 10016, and 4Physiological Science Department, \\ University of California at Los Angeles, Los Angeles, California 90095-1527
}

Null alleles of the mouse Engrailed-2 gene, a molecular homolog of the fly gene engrailed, have demonstrable effects on the anteroposterior $(\mathrm{A} / \mathrm{P})$ patterning of cerebellum as reflected in the disruption of the normal process of foliation of the cerebellar cortex and the alteration of transgene expression boundaries in the adult. Engrailed-2 also affects the transient mediolateral (M/L) pattern of $E n-1$ and $W n t-7 b$ expression seen in late embryogenesis. We have examined three markers of cerebellar compartmentation in En-2 mutant mice: the Zebrin II and Ppath monoclonal antibodies and the transgene L7lacZ. In En-2 mutants, the normal temporal pattern of expression is preserved for all three markers, although the size and spatial location of various bands differ from those of the wild type. Unlike the foliation abnormalities, the $\mathrm{M} / \mathrm{L}$ pattern disturbances we have found occur in nearly all cerebellar regions. Cell counts reveal that all major cell types of the olivocerebellar circuit are reduced by $30-40 \%$. We propose that these results are best explained by a model in which the Engrailed-2 gene is involved in the early specification of the cerebellar field including the number of progenitors. Because each of these progenitors gives rise to a clone of defined size, Engrailed-2 helps specify adult cell number. We further postulate that the configuration of the seven Zebrin bands as well as the shapes and locations of the cerebellar lobules are set up by a second patterning event that occurs after neurogenesis is complete.

Key words: Purkinje cell; deep cerebellar nuclei; inferior olive; L7; Zebrin; compartments
A fundamental characteristic of the cerebellum in all vertebrates is its regionalization into zones with distinct biochemical, genetic, and physiological traits (Wassef et al., 1985, 1987, 1992; Hawkes and Leclerc, 1987; Oberdick et al., 1990, 1993; Hawkes and Gravel, 1991; Millen et al., 1995; Hawkes and Herrup, 1996) The properties of these zones meet many of the criteria necessary to define them as compartments: sharp boundaries, restricted gene expression, and barriers to cell movement (for a complete discussion, see Herrup and Kuemerle, 1997). In the present study we explore the distortion of the murine cerebellar compartments in the Engrailed-2 mutant mouse.

Two homologs of the Drosophila engrailed gene exist in the mouse. Engrailed expression is found in a strong transverse band at the early midbrain-hindbrain junction with decreasing levels toward the rostral midbrain. Consistent with the En gene being essential for brain development, a null allele of mouse Engrailed-1 has been shown to lead to the absence of most of the cerebellum and midbrain (Wurst et al., 1994). The second engrailed homolog, Engrailed-2 (En-2), is also expressed in the presumptive midbrain-hindbrain region. Although En-1 expression initiates at the

Received May 2, 1997; revised July 31, 1997; accepted Aug. 6, 1997.

This work was supported by National Institutes of Health Grant NS 18381 to K.H. We express our thanks to Drs. John Oberdick, Richard Smeyne, and James Morgan for the transgenic mice used as part of this study.

Correspondence should be addressed to Karl Herrup, Alzheimer Research Laboratory, Case Western Reserve Medical School, 10900 Euclid Avenue, Cleveland, $\mathrm{OH} 44106$.

Copyright (C) 1997 Society for Neuroscience $\quad 0270-6474 / 97 / 177881-09 \$ 05.00 / 0$ one-somite stage (McMahon et al., 1992), En-2 expression is not detected until the five-somite stage (Davis and Joyner, 1988; Davis et al., 1988). Unlike En-1, null alleles of Engrailed-2 have only a subtle neurological phenotype (Gerlai et al., 1995), and a modest yet reproducible disruption in the anteroposterior $(\mathrm{A} / \mathrm{P})$ pattern of cerebellar foliation and transgene expression (Joyner et al., 1991; Millen et al., 1994). In addition, mediolateral (M/L) pattern changes have also been noted. There is a subtle change in the banded organization of spinal cord mossy fiber afferents (Vogel et al., 1996) and in the transient pattern of banded expression of $E n-2, E n-1, P a x-2$, and $W n t-7 B$ seen during late gestation (Millen et al., 1995).

In the present study we have used three $\mathrm{M} / \mathrm{L}$ markers: Zebrin II, Ppath, and the expression of an L7lacZ fusion gene. The Zebrin II monoclonal antibody defines subsets of adult Purkinje cells that are arranged in a series of evolutionarily conserved, antigen-positive, sagittal stripes (Hawkes and Gravel, 1991; Hawkes and Herrup, 1996; Hawkes and Eisenman, 1997). There are three Zebrin bands per hemicerebellum in the vermis, a fourth one at the margin of the vermis and hemispheres, and three more in each hemisphere. The pattern is mirror symmetric around the midline and can be recognized in a broad range of adult vertebrates. The borders of the bands have been shown to be congruent with the segregation of some cerebellar afferent terminal fields (Gravel and Hawkes, 1987, 1990), emphasizing the functional significance of the banding pattern. A second marker, the Ppath antigen, reveals a nearly complementary pattern (Leclerc et al., 1992). These reagents are only useful in the adult, 
however, because before postnatal day 6 (P6) there is no Zebrin staining at all, and it is not until P25 that the mature banding pattern is fully developed (Leclerc et al., 1988). In the neonate, $\mathrm{M} / \mathrm{L}$ organization can be visualized using the L 7lacZ transgene of Oberdick et al. (1990, 1993). With this marker, a transient pattern of bands first appears at the midline around embryonic day 18 (E18), with additional bands added laterally throughout postnatal development until about P11, when all Purkinje cells turn positive for transgene expression.

The present study is a comparison of the Zebrin II, Ppath, and LlacZ bands in the wild-type and $E n-2^{\text {hd/hd }}$ cerebellum. Although distinct patterning changes are observed with all three markers, the basic neonatal and adult banding structure is well preserved. In addition to these qualitative studies, we have performed cell counts of four cell populations in the olivocerebellar circuit. We find that the numbers of Purkinje, granule, deep nuclear, and inferior olivary neurons are reduced in the En-2 mutant, each by the same amount. The results are incorporated into a new model of cerebellar development in which cerebellar space undergoes two separate patterning events during ontogeny.

\section{MATERIALS AND METHODS}

Mouse strains. Mice carrying the $E n-2^{\text {hd }}$ allele (Joyner et al., 1991) were maintained on a $129 / \mathrm{Sv}$ inbred background by breeding homozygotes to either heterozygous or homozygous animals. Offspring were genotyped via PCR with the following primers: (1) TTGAGAAGAGAGGCCCTGTA, a sequence common to both $+/+$ and $E n-2^{\text {hd }} / E n-2^{\text {hd }}$ animals; (2) CTCGAACAAAAGGCCAGTGT, a sequence specific for the $+/+E n-2$ homeobox; and (3) TCTCATGCTGGAGTTCTTCG, a sequence in the neomycin gene in the $E n-2^{\text {hd }}$ mutation.

In wild-type animals, these primers amplify a 500-bp band; in homozygous mutants they amplify a 300-bp band; in heterozygotes they amplify one band of each size. Because we could detect no cytoarchitectural differences in their cerebellums, wild-type and heterozygous $\left(E n-2^{h d /+}\right)$ mice were used interchangeably as normal controls in all studies except for the cell counts, in which $+/+$ controls were used exclusively. Mice bearing the L7lacZ transgene were a generous gift from Drs. Richard Smeyne, (St. Jude Research Hospital, Memphis, TN), John Oberdick (Ohio State University, Columbus, OH), and James Morgan, (St. Jude Research Hospital, Memphis, TN). This line derives from a chimeric gene in which the bacterial lacZ ( $\beta$-galactosidase) histological marker is driven by $4 \mathrm{~kb}$ of promoter sequence from the Purkinje cell-specific $L 7$ gene. Originally developed in mice of the B6SJL genetic background, our colony was maintained by brother-sister mating before being bred onto the $129 / \mathrm{Sv}-E n-2^{\text {hd }}$ background. All animals were maintained in the American Association for the Accreditation of Laboratory Animal Careaccredited Case Western Reserve University animal facility, where they were maintained on a 14/10 hr light/dark cycle. Food and water were available ad libitum.

Histology. Adult mice were anesthetized with Avertin $(0.025 \mathrm{ml} / \mathrm{gm}$ body weight) and transcardially perfused with PBS for $2 \mathrm{~min}$, followed by $10 \mathrm{~min}$ of $4 \%$ paraformaldehyde in $0.1 \mathrm{M}$ phosphate buffer. Dissected brains were placed in fixative for $6 \mathrm{hr}$ at $4^{\circ} \mathrm{C}$ and then rinsed with $18 \%$ sucrose in PBS for 30 min two times before being placed in fresh sucrose solution overnight. Brains used for Zebrin II and Ppath analysis were sectioned either horizontally or coronally at $40 \mu \mathrm{m}$ using a vibratome or sectioned at $10 \mu \mathrm{m}$ using a cryostat. Postnatal animals were anesthetized with ether before perfusion and brain dissection.

Detection of $\beta$-galactosidase activity. Whole brains and cryostat sections of animals carrying the L7lacZ transgene were stained for $\beta$ galactosidase activity using standard methods. Samples were kept in 5-bromo-4-chloro-3-indolyl- $\beta$-D-galactoside (X-gal) $\operatorname{mix}: 35 \mathrm{~mm}$ $\mathrm{K}_{3} \mathrm{Fe}(\mathrm{CN})_{6}, 35 \mathrm{~mm} \mathrm{~K} \mathrm{~K}_{4} \mathrm{Fe}(\mathrm{CN})_{6} \cdot 3 \mathrm{H}_{2} \mathrm{O}, 2 \mathrm{mM} \mathrm{MgCl}_{2}, 0.01 \%$ sodium desoxycholate, $0.02 \% \mathrm{NP}-40$, and $1 \mathrm{mg} / \mathrm{ml} \mathrm{X-gal,} \mathrm{for} 8-12 \mathrm{hr}$ at $37^{\circ} \mathrm{C}$ and then rinsed in PBS and stored in a $0.2 \mathrm{M}$ EDTA-PBS solution at $4^{\circ} \mathrm{C}$. The data shown are representative of three animals at each age. Intermediate ages (e.g., P3, P5, and P12) were also examined but are not shown.

Immunohistochemistry. Floating sections $(40 \mu \mathrm{m})$ were treated in standard 24 well tissue culture plates. Sections were washed three times in Tris-buffered saline (TBS) for 5 min each and then incubated in blocking solution (TBS containing $10 \%$ goat serum and $0.1 \%$ Triton $\mathrm{X}-100$ ) to prevent nonspecific antibody binding. After $30 \mathrm{~min}$ the sections were reacted simultaneously with the monoclonal antibodies Zebrin II (a gift from Dr. Richard Hawkes, University of Calgary, Calgary, Alberta, Canada) and Ppath (a gift from Drs. Gerald Schwarting and Miyuki Yamamoto, E.K. Shriver Center, Waltham, MA) diluted 1:8 in blocking solution overnight at $4{ }^{\circ} \mathrm{C}$. Sections were then washed four times for $5 \mathrm{~min}$ each in TBS and placed in $10 \%$ goat serum/0.2\% Triton X-100 in TBS for $30 \mathrm{~min}$. Secondary antibodies were then applied for $2 \mathrm{hr}$ at room temperature. For the Zebrin II antibody, FITC-conjugated goat antimouse IgG was used (1:200 in blocking solution). For Ppath, the secondary was Texas Red-conjugated goat anti-mouse IgM (1:400 in blocking solution). Both secondary antibodies were purchased from Jackson ImmunoResearch (West Grove, PA). Secondary antibodies were then removed by rinsing the sections for $20 \mathrm{~min}$ total in TBS, after which the sections were mounted on gelatinized slides and coverslipped in 1:1 TBS-glycerol. In some experiments, Zebrin II was revealed using HRPconjugated goat anti-mouse IgG (Jackson ImmunoResearch) diluted 1:50 in blocking solution. After the normal rinse, the presence of the HRP was revealed by a 5 min incubation with diaminobenzidine in TBS $(0.25$ $\mathrm{mg} / \mathrm{ml}$ ). The observations presented are based on data obtained from at least three animals of each genotype.

Cell counts. Purkinje, granule, inferior olive, deep cerebellar nucleus, and facial nucleus neurons were counted by previously described methods (Herrup and Sunter, 1987; Maricich et al., 1997). For each cell type, a complete set of sagittal or coronal sections $(10 \mu \mathrm{m})$ were identified, and $10-15$ well spaced sections were selected. Two animals of each genotype were used, and all counts were done with cresyl violet-stained material. The nucleus of the cell was used as a criterion for scoring a cell as present. The total numbers of counted cells for the Purkinje cell, olive, deep nuclear, and facial nucleus populations were then determined by graphing the total counts per section as a function of distance from the first cell (or, for Purkinje cells, distance from the midline). The area under this curve was computed to give the uncorrected counts.

For cerebellar granule cells, six fields, $50 \mu \mathrm{m}$ on a side, were selected (at low magnification to reduce bias). Two fields each, chosen from the tops, sides, and bottoms of folia, were counted in each of six sections from across the mediolateral extent of the cerebellum. The average number of cells in these 36 fields was used as the average density of granule cells in a $50 \times 50 \times 10 \mu \mathrm{m}$ region of the counted cerebellum. This density figure was then multiplied by the total volume of the internal granule cell layer (IGL), determined by measuring the area of the IGL in each of 15 well spaced sections. A graph of granule cell layer area as a function of distance from the midline was made, and the area under the curve was computed to give the volume of the IGL. All neuronal counts were corrected for the split-cell error by the method of Hendry (1976).

\section{RESULTS}

\section{Sagittal compartmentation is disrupted in specific regions of the adult cerebellum}

The mice used in this study were maintained on an inbred 129/Sv genetic background (unless otherwise noted). The effects of the $E n-2^{\text {hd/hd }}$ genotype on cerebellar size and the pattern of foliation are reviewed in the sagittal sections shown in Figure 1 (also see (Joyner et al., 1991; Millen et al., 1994). In both vermis and hemisphere, the cross-sectional area of the mutant (Fig. $1 B, D)$ is much reduced from that of the wild type (Fig. $1 A, C$ ). In the vermis of the mutant (Fig. $1 B$ ), the foliation has a relatively normal appearance in anterior cerebellum (lobules I-V) compared with wild type (Fig. 1A). In posterior cerebellum (lobules VI-IX), however, there is a subtle distortion of the foliation pattern; nodulus (lobule $\mathrm{X}$ ) seems unaffected. In the hemispheres, the anterior lobules of the mutant are well preserved (Fig. 1D) compared with wild type (Fig. 1C), but there is a fusion of Crus II and the paramedian lobule (pml).

We have reconstructed the pattern of Zebrin II and Ppath bands in adult animals using both coronal and horizontal serial sections. The analysis was performed both at low magnification to score the overall pattern of bands and at high magnification to score the phenotype of individual Purkinje cells more accurately. We based our comparisons on the nomenclature established 

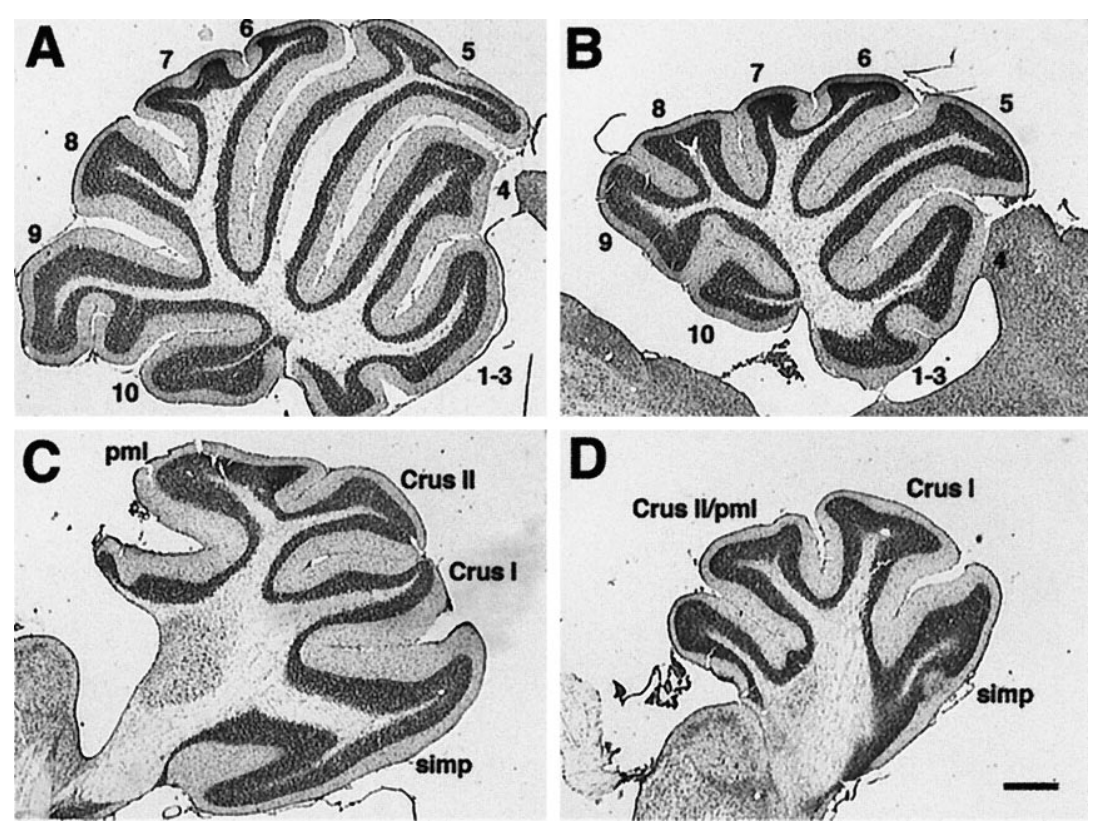

Figure 1. Sagittal view of wild-type and En-2 mutant cerebella cresyl violet-stained midline $(A, B)$ and lateral $(C, D)$ sections from wild-type $(A, C)$ and $E n-2^{h d / h d}(B, D)$ animals. Note the posterior folial abnormalities in lobules 8 and 9 at the midline (compare $B$ with $A$ ) and the fusion of the Crus II and pml lobules in the hemisphere (compare $D$ with $C$ ). Scale bar, $500 \mu \mathrm{m}$. pml, Paramedian lobule; simp, simplex.
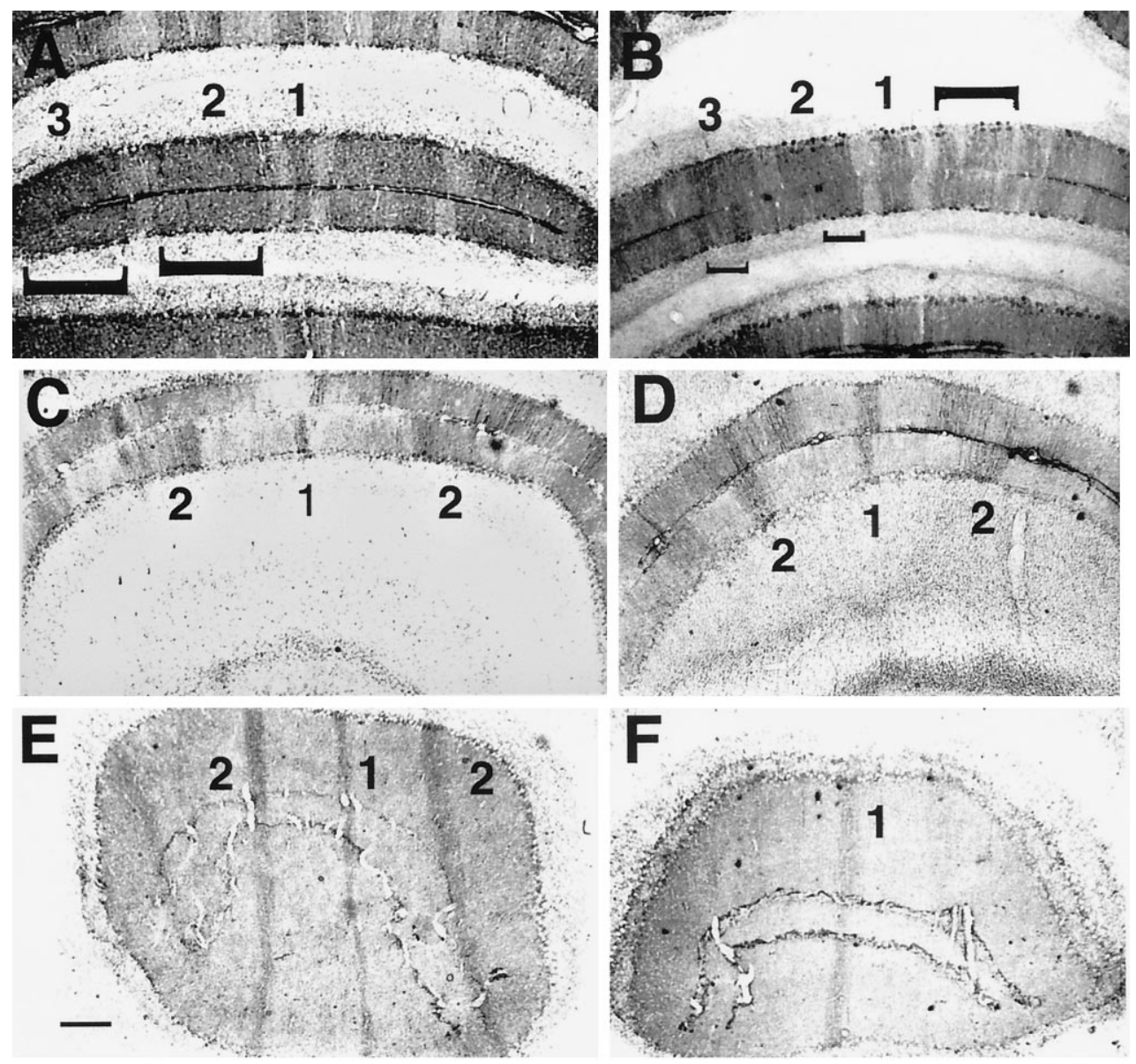

Figure 2. Lack of $E n-2$ function results in the fragmentation or loss of Zebrin II bands in the adult. Zebrin II bands in wild-type $(A, C, E)$ and $E n-2^{\text {hd/hd }}(B, D, F)$ cerebellar cortex are identified according to the nomenclature of Hawkes and Gravel, 1991. Sections are from posterior lobules VIII and IX $(A, B)$, dorsal lobule $\mathrm{V}(C, D)$, and anterior lobule III $(E, F)$. Note the shortening of the mutant $\mathrm{P} 2$ and P3 bands in the posterior lobules on the left (up-facing brackets in $A, B$ ) and the fragmentation of the same bands on the right (down-facing bracket). In lobule $\mathrm{V}$ $(C, D)$ there is a similar shortening of the P2 bands bilaterally, whereas in lobule III $(E, F)$ the $\mathrm{P} 2$ band is missing in the mutant. Scale bar, $200 \mu \mathrm{m}$.

previously (Hawkes and Leclerc, 1987; Millen et al., 1994) The pattern of Zebrin II and Ppath expression is basically intact in the adult $E n-2^{\text {hd/hd }}$ mouse, yet there are significant and reproducible differences between mutant and wild-type Zebrin II staining.

In the cerebellum as a whole, we have identified four regions in which the Zebrin II $\mathrm{M} / \mathrm{L}$ pattern is affected by the $E n-2^{\text {hd }}$ mutation. In each of these regions there is a localized loss or fragmentation of bands (Fig. 2). The discrete nature of the disruptions is highlighted by the fact that a normal banding pattern is often observed in regions immediately adjacent to the disrupted area on a single section. Two of the affected areas are in the vermis: the area that includes the misshapen lobules VIII 


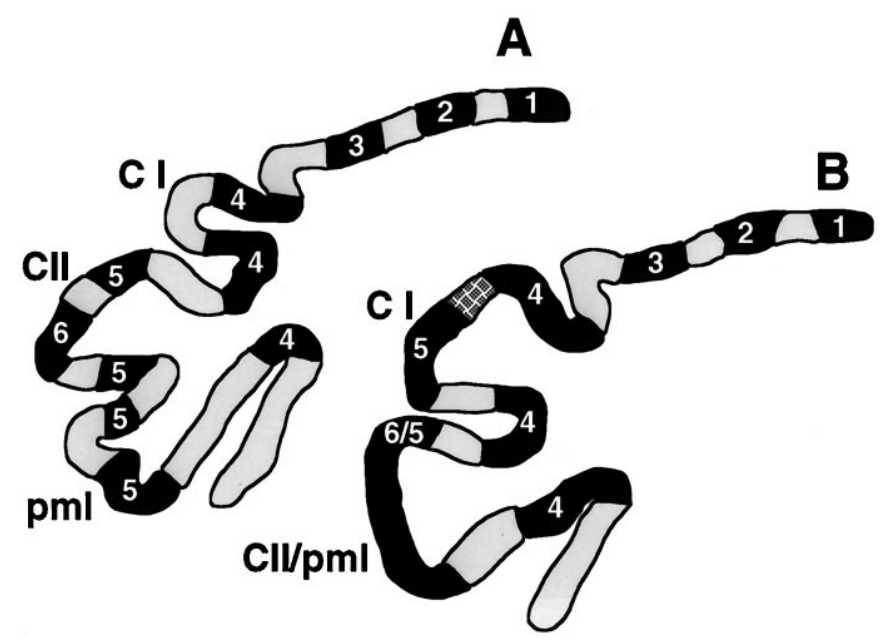

Figure 3. Reconstruction of coronal sections of Zebrin II- and Ppathstained cerebellar cortex. The relative locations of the Zebrin II bands (filled lines) and Ppath interbands (stippled lines), as well as the folial differences between wild type $(A)$ and the $E n-2^{\text {hd/hd }}$ mutant $(B)$ are illustrated in a representative coronal section. The hatched region in the mutant Crus I ( $C I$ ) folium represents Purkinje cells reactive to both Zebrin II and Ppath. The Ppath-positive interband between Zebrin II bands P5 and P6 is either missing or the cells present in this region have switched from Ppath to Zebrin II expression. CII, Crus II.

and IX (Fig. $2 A, B$ ) and the more anterior region encompassing lobule V (culmen; Fig. 2C,D). Each of these shows a fragmentation of bands (see the regions highlighted by brackets). In the third region, lobule IV (centralis), the pattern disruption is evidenced by band loss (Fig. 2E,F). In this more anterior region, the P2 band (nomenclature of Hawkes and Leclerc, 1987) is specifically affected, whereas the midline, $\mathrm{P} 1$, band is always recognizable and largely unaltered. Likewise, in both the mutant and wild type, the P4 Zebrin II band, which occurs at the junction between the vermis and the hemispheres, is always intact. These last two observations suggest that the attenuations noted in the other bands are not simply attributable to a reduced number of Purkinje cells in the cerebellum as a whole. The fourth disruption occurs in the hemisphere in the region of fusion between the Crus II and pml lobules, as well as in the more anterior Crus I lobule (Fig. 3). In these locations, the Zebrin and Ppath patterns are not maintained, and although the exact nature of the disruption is varied, there appears to be a consistent loss of the Ppath-positive interband between the Zebrin II-positive bands, P5 and P6. The result is a continuous Zebrin II-positive band along the crown of the fused folia. Additionally, in some animals, Ppath-positive cells are apparent but misplaced.

In the remaining regions of the cerebellum, there are at most only minor alterations in the Zebrin II and Ppath staining. The banding in the structurally unaffected hemispheric folia of the mutants is generally indistinguishable from that of the wild type, although it appears as if the patterns may not always be bilaterally symmetric. This is most noticeable at the junctions between folia; the base of a specific fissure may be positive for Zebrin II on one side but positive for Ppath (and negative for Zebrin II) on the other side. Furthermore, in the structurally "normal" lobules (i.e., simplex and pyramis), bands 5-7 are appropriately positioned.

\section{Patterning revealed by the $L$ Ilac $Z$ transgene is disrupted in perinatal En-2 mutants}

The results from the $E n-2^{\text {hd/hd }}$ cerebellum led us to consider whether we could establish a link between this adult pattern and the disruption in the embryonic M/L pattern observed by Millen et al. (1995). Oberdick et al. (1993) have shown that a $\beta$ galactosidase transgene, driven by the promoter of a Purkinje cell-specific protein L7, is expressed in a transient pattern of sagittally oriented bands (interleaved with unstained interbands). Expression begins on approximately E17. We have bred the $L 7 l a c Z$ transgene onto the $E n-2^{\text {hd }}$ background and have analyzed expression patterns in perinatal $+/+, E n-2^{h d /+}$, and $E n-2^{h d / h d}$ littermates to determine the effect, if any, of the En-2 mutation on the early $\mathrm{M} / \mathrm{L}$ organization. No differences could be detected between the patterns of wild-type and heterozygous mice, so the findings from these two types of animals are pooled.

At E18, the staining patterns of $E n-2^{\text {hd/hd }}$ mice (Fig. $4 B$ ) are essentially identical to those of normal mice (Fig. 4A). Two stained patches are observed on either side of the midline on the dorsal surface of the newly developing cerebellum, the region that will give rise to lobules 5-7. On the day of birth $($ E19 = P0), these patches persist dorsally, but differences become apparent on the ventromedial surface where lobules 8 and 9 are developing. In control animals (Fig. 4C), one broad band and one thin band are noticeable on either side of the midline. Although $\beta$-galactosidase activity is visible in the corresponding region in the mutants (Fig. $4 D$, asterisk) it is diffuse, and no discrete bands comparable to wild type (Fig. 4C, 1-4) are visible. This difference is more readily recognizable at later stages (Fig. 4G,H) at which the control animals have four bands total, and the mutant animals have two thin bands at the midline, accompanied by a thick band and a thin band on either side. At P2, the cerebellar midline of control mice is clearly demarcated by a band of unstained tissue (Fig. 4E, asterisk, $G$ ). This is in contrast to the En-2-deficient littermates, in which no such "interband" exists (Fig. 4F,H, asterisks). There is also a precocious appearance of a band in the region of the Crus I lobule of the hemispheres. This band, which is located on the right under the $I$ in Figure $4 F$ (and on the left, to the left of the 6), is not found in control animals of this age. Mutants also exhibit irregularly shaped broad bands in lobule V II (Fig. $4 F$ ) and lack a patch of staining localized to either side of these bands (Fig. 4E,F). The eighth folium has a rather uniform appearance from the midline laterally (Fig. $4 H$ ), whereas the wild type has two clear bands (Fig. $4 G, a, b$ ). Caudally, in the ninth folium, the mutant harbors bilateral curved stripes medially (Fig. $4 H$, band $a$ ), not found in controls (Fig. $4 G$ ), plus two stripes lateral to this (Fig. $4 H, b, c$ ), in contrast to the uniform appearance of the control (Fig. $4 G$ ). In both genotypes, $\beta$-galactosidase staining is strongest in the developing parafloccular regions and in posterior lobules 9 and 10.

By P4 the Purkinje cell banding patterns expand laterally in both normal and mutant cerebella. In addition to the dissimilarities observed in the P2 brains, there is a clear lack of striping in anterior lobules 3 and 4 in the mutant (Fig. 5B, asterisk) compared with controls (Fig. 5A, asterisk). At $\mathrm{P} 7$ striping in the hemispheric lobules is easily recognizable. Bands in the mutant are attenuated in the hemisphere, in the precise region where previous studies have shown a foliation abnormality is developing: a failure of formation of the fissure separating Crus II and the pml (Fig. 5C,D, asterisks). Control P30 mice have at least four distinct stripes in vermal lobules 4-6 (Fig. 5E). In En-2-deficient mice at this age, there are only two broad bands (Fig. $5 F$ ), instead of four thin bands (Fig. 5E), in lobules 4-6. The pattern of transgene expression in P12 and P17 mice is similar to that found in P30 mice (data not shown). In contrast to the originally reported behavior of the transgene, a striped appearance of the cerebellar staining persists 

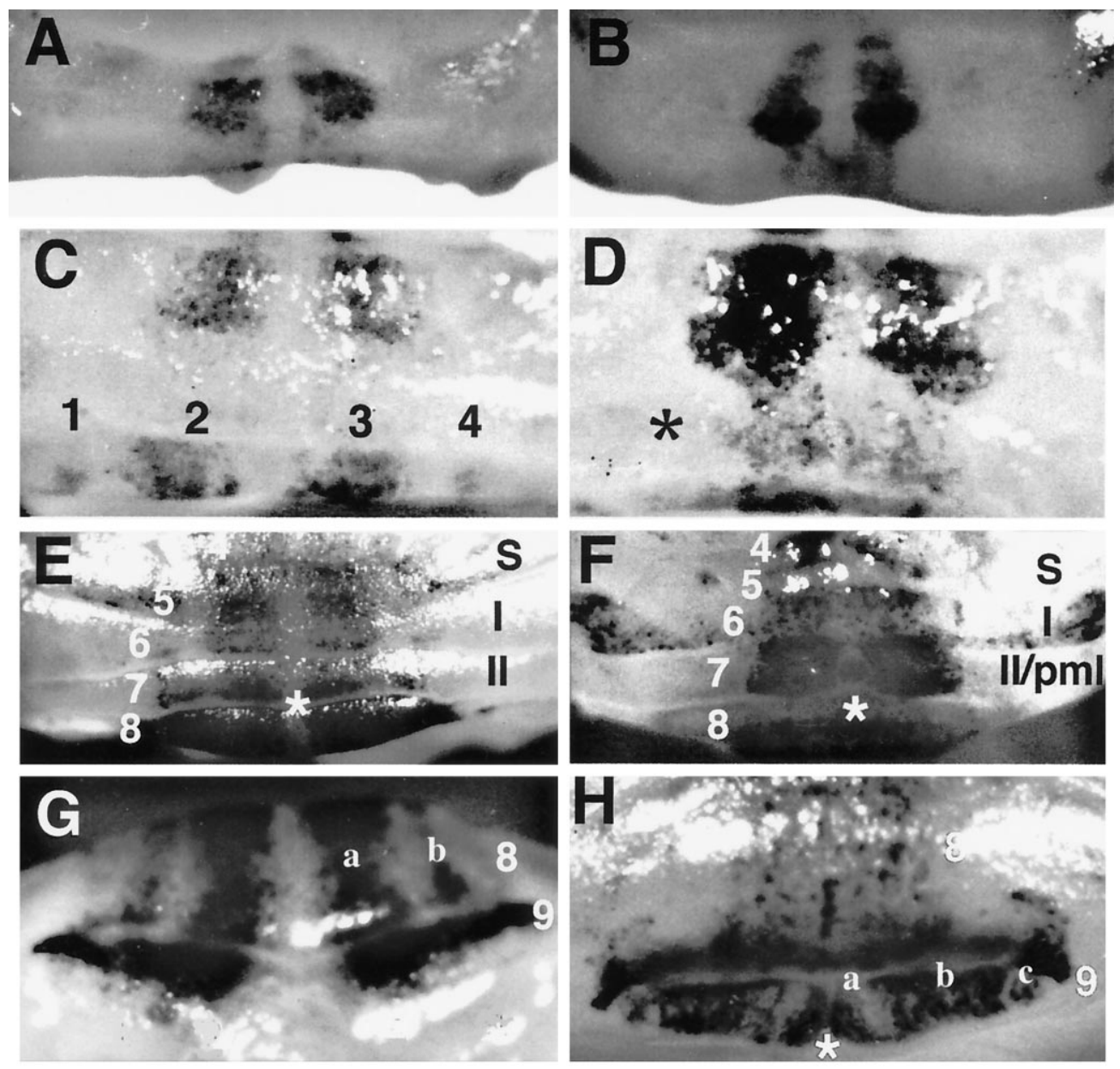

Figure 4. Developmental compartments are disrupted early in the $E n-2^{\text {hd/hd }} \mathrm{mu}-$ tant. Whole-mount cerebella from $E n$ $2^{\text {hd/hd }}$, LlacZ $(B, D, F, H)$ stained with $\beta$-galactosidase are compared with wildtype mice $(A, C, E, G)$ at different perinatal ages: $\mathrm{E} 18(A, B), \mathrm{P} 0(C, D)$, and $\mathrm{P} 2$ $(E, F)$. At E18, two medial bands are present in both control $(A)$ and mutant $(B)$ animals. By $\mathrm{P} 0$, the first disruptions are apparent. In wild-type $(C)$, four bands are located in the region of the eighth and ninth folia, compared with the highly diffuse staining (asterisk) found in Engrailed-2 mutants (D). At P2 $(E-H)$ many differences are noticeable. There is a loss of a midline interband ( $F$, asterisk), the precocious appearance of a stained band in Crus I $(F$, located under $I$ in the mutant), and the addition of two medial bands in lobule IX $(G$, compare bands $a$, $b$ in wild type with the pattern in the En-2 $2^{\text {hd/hd }}$ lobules 8, 9, H). S, Simplex. in both controls and mutants even at P30. Because of this, the differences in the striping pattern of the L7lacZ transgene in normal and mutant animals remain apparent. The reason for the persistence of the striped pattern is unknown; it may be attributable to quantitative variations in expression (all Purkinje cells express, but some much less than others); it may reflect an alteration in the transgene structure with continued germ line transmission; or it may be the effects of the $129 / \mathrm{Sv}$ genetic background.

\section{Purkinje cell identity may be affected by the $E n-2^{\text {hd }}$ mutation}

LeClerc et al. (1990) established the existence of a binary Zebrin and Ppath code that partitions adult Purkinje cells into one of three phenotypes. Most Purkinje cells fall into two major classes: Zebrin II-positive, Ppath-negative and vice versa. A third, minor class is found in the P3 band in lobule IX, in which the Purkinje cells are positive for both markers. LeClerc et al. (1992) also noted that Purkinje cells at the interface of Zebrin II and Ppath bands were often double-labeled. In the Engrailed-2 mutant, most regions retained the standard code; Purkinje cells were either Zebrin II- or Ppath-positive, with double-labeled cells found primarily in the P3 band in the rostral portion of posterior lobe IX. This situation persisted, even in regions where the bands were lost or fragmented. However, in many mutant animals, doublestained cells replaced the usual Ppath-positive interband located at the crown of Crus I (between Zebrin-positive bands 4 and 5; Fig. $3 A, B)$. Thus, it appears as if the identities of individual
Purkinje cells were altered at this one location. One possibility is that the Purkinje cells in this double-labeled band were transformed into cells resembling those in the P3 band in lobule IX; a second possibility is that this region represents an expanded or ambiguous "boundary" between a Zebrin and Ppath band. In neither the mutant nor the wild type could we identify any double-negative cells.

\section{Cell numbers in the En-2 ${ }^{\text {hd/hd }}$ olivocerebellar circuit}

The reduced size of the cerebellum in En- $2^{\text {hd/hd }}$ mutant animals has been described previously, but the implied deficit of cells has only been documented in a preliminary manner (Herrup et al., 1991). Two adult En-2 $2^{\text {hd/hd }}$ animals and two $+/+$ controls were serially sectioned in the sagittal or coronal plane, and the numbers of Purkinje, granule, deep nuclear, and inferior olive cells were counted as described in Materials and Methods. These counts are illustrated graphically in Figure $6 \mathrm{~A}$. Note that each of the cell types involved in the olivocerebellar circuit is reduced in number by $30-40 \%$. Without a careful quantitative study during development, we cannot determine whether the deficit is caused by cell death or agenesis. However, a reduction in cerebellar size in En-2-deficient animals is apparent as early as E15 (AvaradoMallart et al., 1990), and no obvious period of cell death has been observed in our studies. To ensure that the cell number deficit was not a property of the entire CNS of the mutant, we counted the motor neurons of the facial nucleus in both mutant and wild-type animals. Although the cells of this nucleus are located in the 

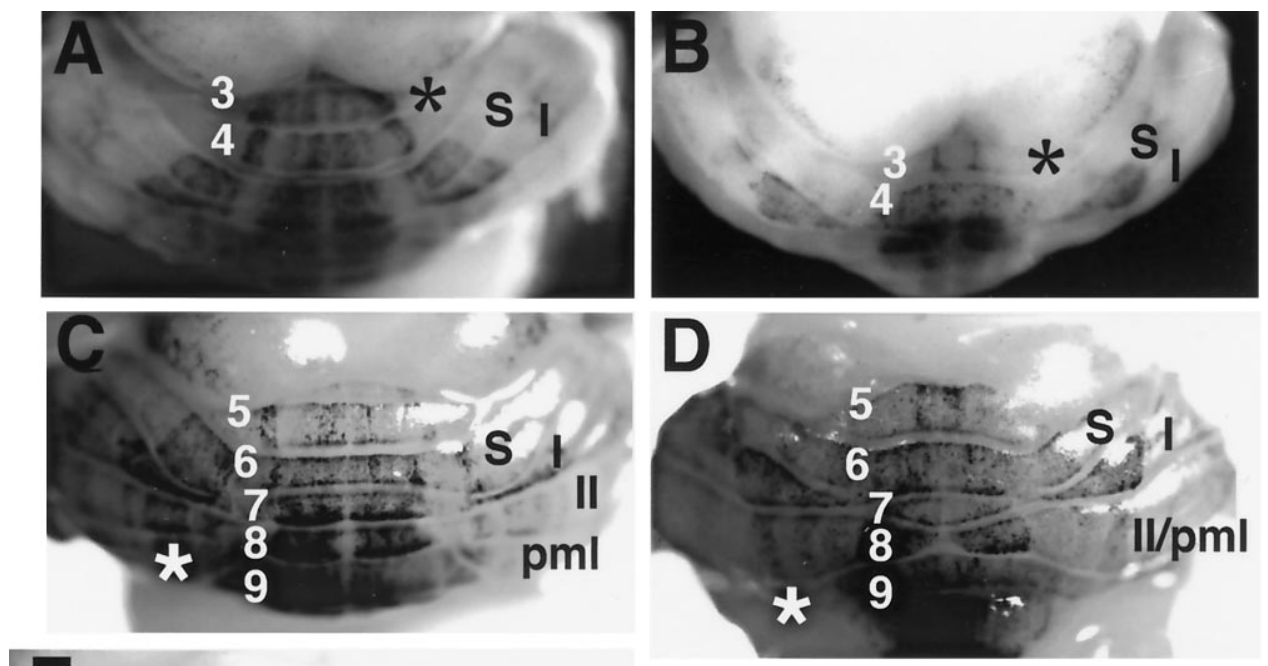

5. LlacZ band loss persists into adulthood. Whole-mount cerebella from $E n-2^{\text {hd/hd }}$, L7lacZ $(B, D, F)$ stained with $\beta$-galactosidase are compared with wildtype mice $(A, C, E)$ at different postnatal ages: $\mathrm{P} 4(A, B), \mathrm{P} 7(C, D)$, and $\mathrm{P} 30(E, F)$. $\mathrm{P} 4$ mutants lack lateral bands of transgene activity in vermal lobules III and IV, and staining is generally diffuse $(A, B$, asterisks). By P7 staining in the Crus II- and pml-fused folia reveals that band width is significantly reduced in the mutant (compare the region identified by the asterisk in $C$, lobule 8 , with that in $D$ ). Severe band loss is recognizable in the adult mutant $(F)$, most noticeably in the vermis compared with wild type $(E)$. In general, there appears to be less overall reaction product in En-2 mutants at this time. $S$, Simplex; I, Crus I; II, Crus II.
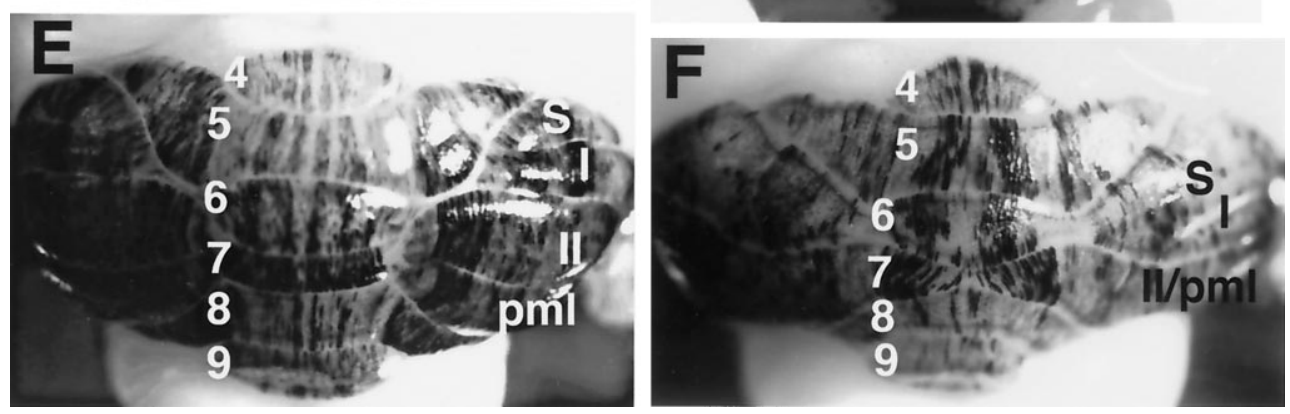

ventral brainstem, $750 \mu \mathrm{m}$ rostral to the inferior olive, the cell counts in mutant and wild-type animals are identical.

The Purkinje cell counts were analyzed in greater detail for evidence of regional variation in either the $\mathrm{M} / \mathrm{L}$ or $\mathrm{A} / \mathrm{P}$ axis. Figure $6 B$ is a display of the raw counts of hemicerebella of two $E n-2^{\text {hd/hd }}$ and two wild-type animals. The reduced width of the mutant structure is reflected in the smaller $x$-intercept, but the overall shapes of the curves are nearly identical. This suggests that there is little or no regional variation in the $\mathrm{M} / \mathrm{L}$ axis. A similar conclusion derives from Figure $6 C$, in which the Purkinje cell counts are displayed folium by folium. Each box in the figure is proportional in area to the number of Purkinje cell counts in that folium. Within each box, the shaded area represents the $E n-2^{\text {hdlhd }}$ counts for that region. From this analysis it would appear that there is a nearly uniform reduction in Purkinje cell number in all $\mathrm{A} / \mathrm{P}$ and $\mathrm{M} / \mathrm{L}$ regions.

\section{DISCUSSION}

The initial goal of this study was to determine whether the alterations in cerebellar size and $\mathrm{A} / \mathrm{P}$ patterning that have been described for the $E n-2^{\text {hd }} / h d$ mouse were accompanied by any effect on the organization of the cerebellar cortex in the $\mathrm{M} / \mathrm{L}$ dimension after birth. This question is of interest because during late embryogenesis, the cells expressing En-2 form a pattern of sagittal bands that divides the E17.5 cerebellum into M/L compartments, presaging the strong orientation of the adult modules of cerebellar function.

We have used three markers to assess the $\mathrm{M} / \mathrm{L}$ patterning of the cerebellum in the En-2 mutants: Zebrin II, Ppath, and the L7lac Z transgene. We find that most of the M/L patterning is maintained, but there are subtle, consistent changes, which are a part of the phenotype of this mutation. Significantly, the disturbances we have found are not exclusively in those regions where the mutation has obvious structural (i.e., folial) abnormalities. Lobules VIII and IX in the posterior vermis are affected in their structure; here there is a correlated disruption of both the L7lacZ and Zebrin II patterns (Figs. $2 A, B, 4 G, H$ ). By contrast, the more anterior lobules, III and IV, appear normal in their lobular structure but show significant alterations in the pattern of both the L7 and Zebrin markers (Figs. 2E,F, 5A,B). These data suggest that the consequences of the En-2 mutation for adult cerebellar structure cover a much broader area than previously appreciated. Curiously, there does not appear to be a discernible correlation between the postnatal pattern of En-2 expression and the location of the L7 or Zebrin changes. For example, at birth En-2 is expressed in a broad band of cells at the midline. The L7 transgene is appropriately expressed dorsally at this time, but in ventral regions, transgene expression is disrupted in mutants. Also, in the early postnatal animal, disruptions revealed by the L7 marker do not always correlate with the disturbances in the adult, as revealed by the Zebrin II and Ppath bands. For example, in the $E n-2^{\text {hd/hd }}$ neonate, there is no clear demarcation of a well defined L7lacZ-negative band at the midline (Fig. $4 E, F$ ), whereas the P1 Zebrin band, which is located precisely at the midline, always remains intact in the adult (Fig. 2).

The embryonic (E17.5) pattern of En-2 expression correlates somewhat better with the $L 7 l a c Z$ changes, but even here there is not perfect congruence. The precocious appearance of the L7lacZ bands in Crus I (at P2) and the ectopic expression of $L 7 l a c Z$ at the midline both correlate with the loss of En-2 expression in the similar regions of the E17.5 embryos. On the other hand, there are regions where the loss of En-2 function at E17 appears to have no comparable gain in L7lacZ expression. Apart from the two abnormalities cited above, it is difficult to attribute any other change in L7lacZ expression specifically to a loss of 
A

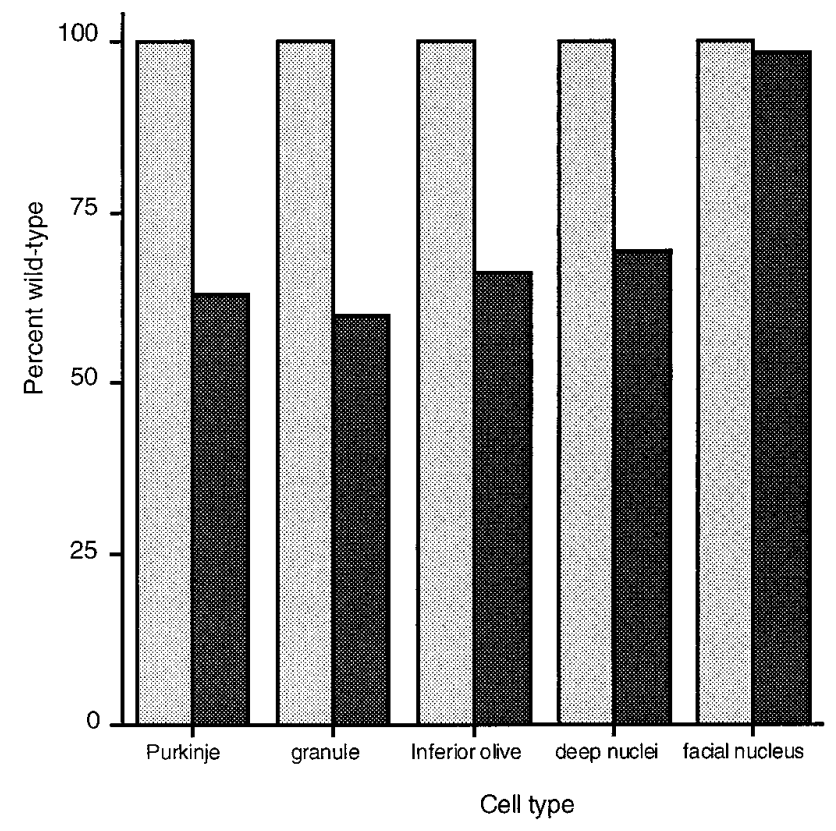

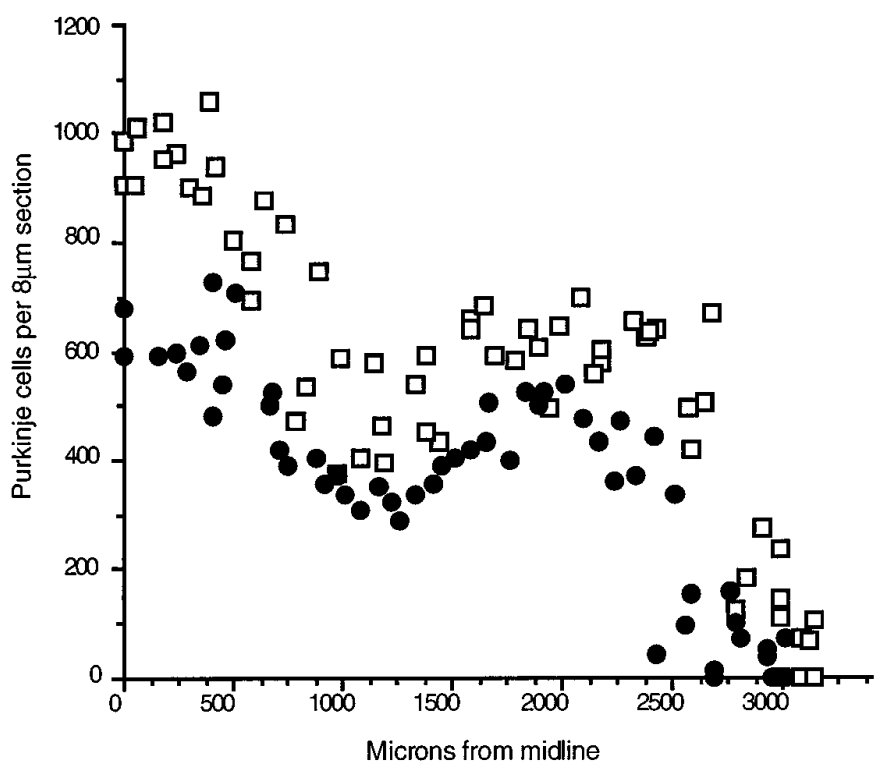
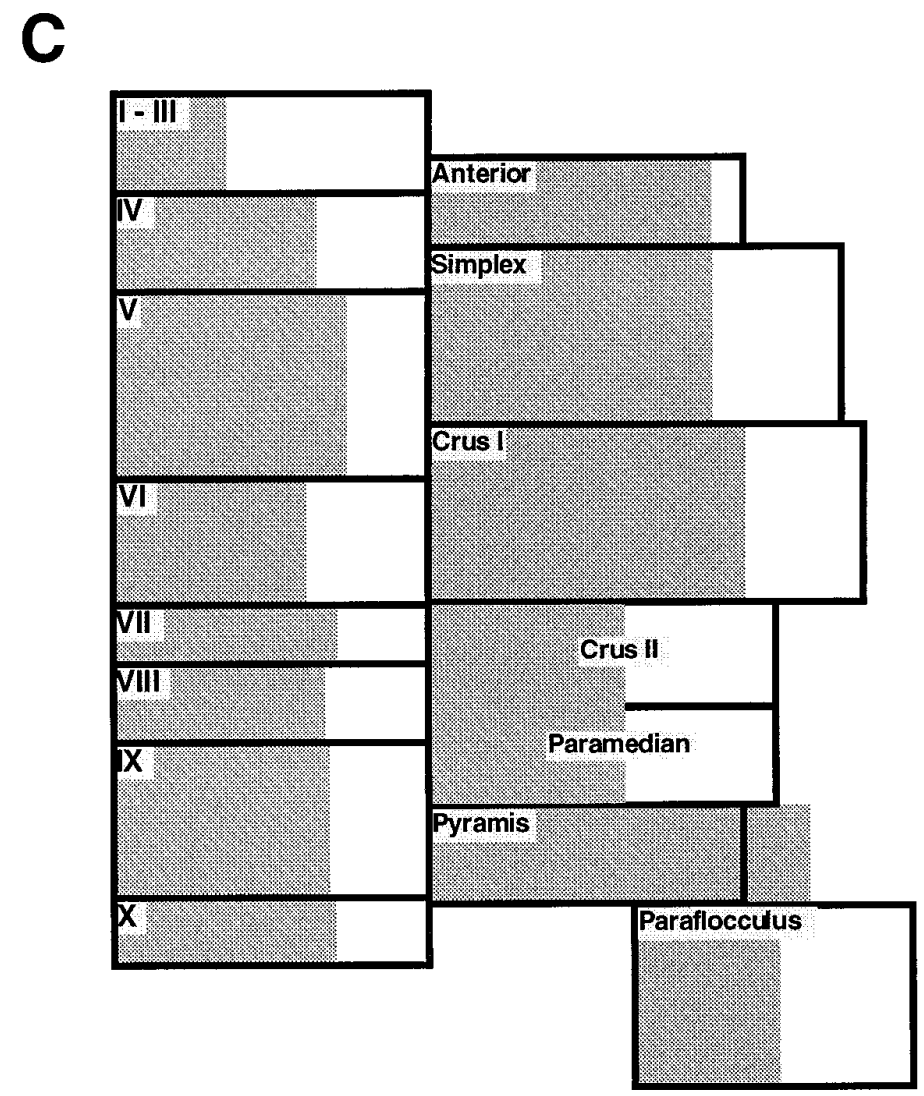

Figure 6. A, Effect of Engrailed-2 on neuronal cell number. The percentage of each cell type in wild-type (shaded bar, 100\%) and affected (solid bar) animals is shown. All of the principal neurons of the olivocerebellar circuit are affected to about the same extent. Counts of the motor neurons of the facial nucleus serve as a control, indicating that the deficit is specific for the cell types shown. $B$, Uniform reduction of Purkinje cell number. The raw counts from hemicerebella of mutant and control animals are shown graphically ( $\square$, wild-type; $\boldsymbol{\bullet}$, En- $2^{\text {hd/hd }}$ ). That the overall shapes of the curves are nearly identical indicates the lack of regional variation in the Purkinje cell loss observed in the mutant. $C$, Purkinje cell loss in the En-2 mutant cerebellum. In this diagram, Purkinje cell counts are displayed folium by folium. Each box is proportional in area to the number of Purkinje cells in the corresponding folium. The shaded region represents the the number of Purkinje cells found in each folium in the En-2 mutant. In each case, about two-thirds of the box is shaded, indicating an approximate one-third loss of cells. This loss appears uniform, because it is not greater or lesser in any given folium. 
En-2 function. The same applies to the Zebrin II and Ppath pattern. Thus although the $\mathrm{M} / \mathrm{L}$ pattern of cerebellar compartmentation is clearly disrupted, the nature of this disruption, in and of itself, does not inform us about the link between the loss of En-2 expression and the observed structural and biochemical consequences.

The other informative phenotype of the En-2 mutant is the absence of neurons in the olivocerebellar circuit. We find that each of the four major cell populations is reduced in En-2 mutant mice, and all four are reduced by a similar amount: $30-40 \%$. To our knowledge, this is the first reported instance of a uniform decrease in these four cell types. The deep nuclei, in particular, have been reported to show cell number deficits in only three other mutations: weaver (Maricich et al., 1997), vibrator (Weimer et al., 1982), and purkinje cell degeneration (Triarhou et al., 1987). Although reductions in olive cell number are known to occur in a number of mutants, they are rarely if ever as large as those found in their cortical targets. For example, the weaver mutant is missing $35 \%$ of its cerebellar Purkinje cells, yet it contains a full complement of olive neurons (Blatt and Eisenman, 1985; Herrup and Trenkner, 1987); lurcher loses $100 \%$ of its Purkinje cells but only $80 \%$ of its olive neurons (Caddy and Biscoe, 1979). As with the biochemical markers discussed previously, there is no obvious correlation between the known expression patterns of the normal Engrailed-2 gene and the location of the missing cells. There is not an augmented loss of Purkinje cells in the vermis, as might be expected from the $L$ lac $Z$ misexpression patterns found in the mutant, and there is no banded organization to the Purkinje cell loss, as is found in the nervous (Edwards et al., 1994) and leaner (Heckroth and Abbott, 1994) mutations. Rather, if the Purkinje cell distribution may be taken as example (Fig. $6 C$ ), there is a near uniform loss of cells from all regions.

\section{A double-patterning hypothesis}

We believe that our data are best explained by a model in which cerebellar space is patterned twice. The first patterning event occurs at E8-E10 when the overlapping but noncongruent bands of En-1 and En-2 expression appear at early neural tube stages. This event establishes the cerebellar field and specifies the precursors that will give rise to the various cells of the cerebellum. We also propose that subdivisions of the cerebellar field are established in this first patterning event. A simple model would be that there are three major subdivisions: En-1-only, En-2-only, and $E n-1$ plus -2. Note that this event occurs at a time before the bulk of neurogenesis has occurred. This view is consistent with earlier observations that the population of adult Purkinje cells descends from a small number of precursors established early in CNS development. Exact estimates of the initial number of precursors vary from 8 to 11 (Herrup and Sunter, 1986) to 64 (Baader et al., 1996) per half-cerebellum, but there is agreement that the event(s) that selects these cells occurs during the early neural tube stage, embryonic days 8-9, the same period of development when Engrailed-1 and Engrailed-2 expression are first observed by in situ hybridization. Based on an analysis of Purkinje cell clone size in several different inbred strains of mouse, the early Purkinje cell precursors are hypothesized to produce clones of cells with a size (measured as the number of Purkinje cells per clone) that is intrinsic to the precursor (Herrup and Sunter, 1986). Thus the initial establishment of the precursors is a central event in the establishment of cell number in the cerebellar system. We propose that in the $E n-2^{\text {hd/hd }}$ mouse, the early establishment of the cerebellar neuronal precursors is blocked (either by death or agenesis). Following with the simple example of three Engrailed compartments, we might predict that the cerebellum of the En$2^{\text {hd/hd }}$ mouse is missing the En-2-only and En-1 plus - 2 cell lineages.

After these early events, we propose that the spatial coherence of the initial subdivisions is lost. Several independent lines of evidence demonstrate that there is extensive spatial mixing of the neuronal cell lineages in the mouse. Direct observation of mixing in the ventricular zone has been reported by Fishell et al. (1993) and in the postmitotic populations by O'Rourke et al. (1995, 1997). Cell lineage studies in aggregation chimeras (Herrup, 1987), X-inactivation mosaics (Baader et al., 1996), and retroviral mosaics (Gray et al., 1988; Walsh and Cepko, 1992, 1993; Reid et al., 1997) have provided abundant evidence that descendants of the early nerve cell precursors intersperse widely with each other within the growing brain. The expectation, therefore, is that the first subdivisions should not stay as spatially defined patches; rather, they should intermingle and spread throughout the adult cerebellum.

Our findings in $E n-2^{\text {hd/hd }}$ mice are consistent with this model. We find that the various cell populations of the cerebellar field are reduced by a nearly identical percentage, even the cells of the inferior olive that migrate to a distant position in the ventral medulla. Furthermore, as predicted by the model, the spatial distribution of the remaining Purkinje cells is nearly uniform across the entire $\mathrm{A} / \mathrm{P}$ and $\mathrm{M} / \mathrm{L}$ expanse of the cerebellum. This is likely to be the result of extensive mixing of the lineages during the development of cerebellar cortex. A similar picture of uniform cell loss is observed in aggregation chimeras of either staggerer or lurcher mutants (Herrup and Sunter, 1987; Vogel and Herrup, 1993) or purkinje cell degeneration (Mullen, 1977).

What then is the explanation for the observed pattern of the Zebrin II, Ppath, and L7 bands in the Engrailed-2 mutant? Our study has documented significant and reproducible differences between the $E n-2^{\text {hd/hd }}$ and wild-type patterns, but in view of the fact that the mutant cerebellum contains one-third fewer cells, the fidelity of the pattern to that of the wild type is impressive. We propose that the similarities are the predicted result of a process in which there is a second cerebellar patterning event that occurs after neurogenesis is complete. It is this second event that determines the size and shape of both the folia and the sagittal bands. The exact timing of the second event is not known, but it is noteworthy that the first appearance of $\mathrm{M} / \mathrm{L}$ or $\mathrm{A} / \mathrm{P}$ differentiation in the cerebellar anlage occurs at around E14.5, just after the birth of the large cerebellar neurons (E10-E13.5).

It has been argued that the $\mathrm{M} / \mathrm{L}$ and $\mathrm{A} / \mathrm{P}$ divisions of cerebellar cortex, revealed by the Zebrin bands and the folia, reflect a true developmental compartmentalization (Herrup and Kuemerle, 1997). We would suggest, however, that these spatial compartments are analogs rather than true homologs of the developmental compartments described in the fruit fly. One important reason for this interpretation is our prediction that the formative events that establish them are likely to occur after cell division (and cell mixing) is completed. The relatively normal appearance of the bands and folia in $E n-2^{h d / h d}$ mice raises the question of what role the Engrailed genes have in directing the second patterning event. Our dual-patterning hypothesis is neutral on the question of whether the descendants of the original $E n$-2-only or the $E n-1+2$ precursors are the same ones that express En-2 during late embryogenesis. It is plausible that En-2 is not part of the patterning mechanism at all but merely part of the "realization" program within the individual cell types. For 
the present, however, this important question must remain unanswered.

\section{REFERENCES}

Avarado-Mallart R-M, Martinez S, Lance-Jones C (1990) Pluripotentiality of the 2 day old avian germinative neuroepithelium. Dev Biol 139:75-88.

Baader S, Schilling M, Rosengarten B, Pretsch W, Teutsch H, Oberdick J, Schilling K (1996) Purkinje cell lineage and the topographic organization of the cerebellar cortex: a view from $\mathrm{X}$ inactivation mosaics. Dev Biol 174:393-406.

Blatt G, Eisenman L (1985) A qualitative and quantitative light microscopic study of the inferior olivary complex of normal, reeler and weaver mutant mice. J Comp Neurol 232:117-128.

Caddy K, Biscoe T (1979) Structural and quantitative studies on the normal $\mathrm{C} 3 \mathrm{H}$ and Lurcher mutant mouse. Philos Trans R Soc Lond [Biol] 287:167-201.

Davis C, Joyner A (1988) Expression patterns of the homeoboxcontaining genes En-1 and En-2 and the protooncogene int-1 diverge during mouse development. Genes Dev 2:1736-1744.

Davis C, Noble-Topham S, Rossant J, Joyner A (1988) Expression of the homeo box-containing gene En-2 delineates a specific region of developing mouse brain. Genes Dev 2:361-371.

Edwards MA, Crandall JE, Leclerc N, Yamamoto M (1994) Effects of nervous mutation on Purkinje cell compartments defined by Zebrin II and 9-O-acetylated gangliosides expression. Neurosci Res 19:167-174.

Fishell G, Mason C, Hatten M (1993) Dispersion of neural progenitors within the germinal zones of the forebrain. Nature 362:636-638.

Gerlai R, Millen K, Fabien K, Herrup K, Joyner A, Roder J (1995) Impaired motor learning performance in cerebellar en-2 mutant mice. Behav Neurosci 110:126-133.

Gravel C, Hawkes R (1987) Neuronal maturation in the normal and hypothyroid rat cerebellar cortex studied with monoclonal antibody MIT-23. J Comp Neurol 258:447-462.

Gravel C, Hawkes R (1990) Parasagittal organization of the rat cerebellar cortex: direct comparison of Purkinje cell compartments and the organization of the spinocerebellar projection. J Comp Neurol 291:79-102.

Gray G, Glover J, Majors J, Sanes J (1988) Radial arrangement of clonally related cells in the chicken optic tectum: lineage analysis with a recombinant retrovirus. Proc Natl Acad Sci USA 85:7356-7360.

Hawkes R, Eisenman L (1997) Stripes and zones: the origins of regionalization of the adult cerebellum. Perspect Dev Neurobiol, in press.

Hawkes R, Gravel C (1991) The modular cerebellum. Prog Neurobiol 36:309-327.

Hawkes R, Herrup K (1996) Aldolase C/Zebrin II and the regionalization of the cerebellum. J Mol Neurosci 6:147-158.

Hawkes R, Leclerc N (1987) Antigenic map of the rat cerebellar cortex: the distribution of parasagittal bands as revealed by monoclonal antiPurkinje cell antibody mabQ113. J Comp Neurol 256:29-41.

Heckroth JA, Abbott LC (1994) Purkinje cell loss from alternating sagittal zones in the cerebellum of leaner mutant mice. Brain Res 658:93-104.

Hendry I (1976) A method to correct adequately for the change in neuronal size when estimating neuroanl numbers after nerve growth factor treatment. J Neurocytol 5:337-349.

Herrup K (1987) Roles of cell lineage in the developing mammalian brain. In: Neural development, current topics in developmental biology (Hunt RK, ed), pp 65-97. New York: Academic.

Herrup K, Kuemerle B (1997) The compartmentalization of the cerebellum. Annu Rev Neurosci 20:61-90.

Herrup K, Sunter K (1986) Lineage dependent and independent control of Purkinje cell number in the mammalian CNS: further quantitative studies of lurcher chimeric mice. Dev Biol 117:417-427.

Herrup K, Sunter K (1987) Numerical matching during cerebellar development: quantitative analysis of granule cell death in staggerer mouse chimeras. J Neurosci 7:829-836.

Herrup K, Trenkner E (1987) Regional differences in cytoarchitecture of the weaver cerebellum suggest a new model for weaver gene action. Neuroscience 23:871-85.

Herrup K, Zanjani H, Millen K, Rossant J, Joyner A (1991) Analysis of cerebellar circuits in Engrailed-2 deficient transgenic mice. Soc Neurosci Abstr 17:634.
Joyner A, Herrup K, Auerbach B, Davis C, Rossant J (1991) Subtle cerebellar phenotype in mice homozygous for a targeted deletion of the En-2 homeobox. Science 251:1239-1243.

Leclerc N, Gravel C, Hawkes R (1988) Development of parasagittal zonation in the rat cerebellar cortex: MabQ113 antigenic bands are created postnatally by the suppression of antigen expression in a subset of Purkinje cells. J Comp Neurol 273:399-420.

Leclerc N, Schwarting G, Herrup K, Hawkes R, Yamamoto M (1992) Compartmentation in mammalian cerebellum: Zebrin II and P-path antibodies define three classes of sagittally organized bands of Purkinje cells. Proc Natl Acad Sci USA 89:5006-5010.

Maricich SM, Soha J, Trenkner E, Herrup K (1997) Failed cell migration and death of cerebellar Purkinje can deep nuclear neurons in the weaver cerebellum. J Neurosci 17:3675-3683.

McMahon A, Joyner A, Bradley A, McMahon J (1992) The midbrainhindbrain phenotype of Wnt-1-/Wnt-1- mice results from stepwise deletion of engrailed-expressing cells by 9.5 days post coitum. Cell 69:581-595.

Millen KJ, Wurst W, Herrup K, Joyner AL (1994) Abnormal embryonic cerebellar development and patterning of postnatal foliation in two mouse Engrailed-2 mutants. Development 120:695-706.

Millen KJ, Hui CC, Joyner AL (1995) A role for En-2 and other murine homologues of Drosophila segment polarity genes in regulating positional information in the developing cerebellum. Development 121:3935-3945.

Mullen RJ (1977) Site of pcd gene action and Purkinje cell mosaicism in cerebella of chimeric mice. Nature 270:245-247.

O'Rourke N, Sullivan D, Kaznowski C, Jacobs A, McConnell S (1995) Tangential migration of neurons in the developing cerebral cortex. Development 121:2165-2176.

O’Rourke N, Chenn A, McConnell S (1997) Postmitotic neurons migrate tangentially in the cortical ventricular zone. Development 124:997-1005.

Oberdick J, Smeyne R, Corbin J, Wurzburger R, Morgan J (1990) Subsets of Purkinje cells and bipolar neurons identified by the $\mathrm{L} 7$ promoter. Soc Neurosci Abstr 16:644.

Oberdick J, Schilling K, Smeyne RJ, Corbin JG, Bocchiaro C, Morgan JI (1993) Control of segment-like patterns of gene expression in the mouse cerebellum. Neuron 10:1007-1018.

Reid C, Tavazoie S, Walsh C (1997) Clonal dispersion and evidence for asymmetric cell division in ferret cortex. Development 124:2441-2461.

Triarhou LC, Norton J, Ghetti B (1987) Anterograde transsynaptic degeneration in the deep cerebellar nuclei of Purkinje cell degeneration ( $p c d$ ) mutant mice. Exp Brain Res 66:577-588.

Vogel M, Herrup K (1993) A theoretical and experimental examination of cell lineage relationships among cerebellar Purkinje cells in the mouse. Dev Biol 156:49-68.

Vogel MW, Ji Z, Millen K, Joyner AL (1996) The Engrailed-2 homeobox gene and patterning of spinocerebellar mossy fiber afferents. Dev Brain Res 96:210-218.

Walsh C, Cepko C (1992) Widespread dispersion of neuronal clones across functional regions of the cerebral cortex. Science 255:434-440.

Walsh C, Cepko C (1993) Widespread dispersion in proliferative layers of cerebral cortex. Nature 362:632-635.

Wassef M, Zanetta J, Brehier A, Sotelo C (1985) Transient biochemical compartmentalization of Purkinje cells during early cerebellar development. Dev Biol 111:129-137.

Wassef M, Sotelo C, Cholley B, Brehier A, Thomasset M (1987) Cerebellar mutations affecting the postnatal survival of Purkinje cells in the mouse disclose a longitudinal pattern of differentially sensitive cells. Dev Biol 124:379-389.

Wassef M, Angaut P, Arsenio-Nunes L, Bourrat F, Sotelo C (1992) Purkinje cell heterogeneity: its role in organizing the topography of the cerebellar cortex connections. In: The cerebellum revisited (Llinas R, Sotelo C, eds), pp 5-21. New York: Springer.

Weimer WR, Lane PW, Sidman RL (1982) Vibrator ( $v b)$ : a spinocerebellar system degeneration with autosomal recessive inheritance in mice. Brain Res 1982:357-364.

Wurst W, Auerbach AB, Joyner AL (1994) Multiple developmental defects in Engrailed-1 mutant mice: an early mid-hindbrain deletion and patterning defects in forelimbs and sternum. Development 120:20652075 . 